\title{
THE SIMULATION OF THE TIME CHARACTERISTICS OF THE PHOTORESISTIVE STRUCTURES BASED ON THE CADMIUM LEAD SULFIDES
}

\author{
I.N. Miroshnikova ${ }^{(a)}$, B.N. Miroshnikov ${ }^{(b)}$, E.V. Zenova ${ }^{(c)}$, M.Y. Presnjakov ${ }^{(d)}$, A.A. Makarov ${ }^{(e)}$ \\ (a),(b),(c),(e) National Research University “Moscow Power Engineering Institute”, Moscow, Russia \\ (a),(b),(c) Institute of Nanotechnology of Microelectronics of the Russian Academy of Science, Moscow, Russia \\ (d) National Research Center "Kurchatov Institute", Moscow, Russia \\ ${ }^{(e)}$ International Laboratory of Statistics of Stochastic Processes and Quantitative Finance of the National Research Tomsk \\ State University, Tomsk, Russia

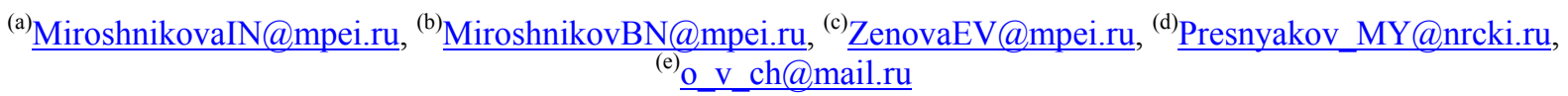

\begin{abstract}
The structures of the lead sulphide and lead selenide based photoresistors are examined. The simulation allowed us to explain the experimentally observed differences in the photoconductivity relaxation time between two different types of photoresistors.
\end{abstract}

Keywords: photoresistor, lead chalcogenides, $\mathrm{Pb}_{x} \mathrm{Cd}_{y} \mathrm{~S}-$ based structures, carrier lifetime, relaxation time

\section{INTRODUCTION}

Photoresistors (PRs) on the base of lead chalcogenides ( $\mathrm{PbS}, \mathrm{PbSe}, \mathrm{PbTe})$ have found widespread applications in infrared (1-5 microns) impulse optical-electronic systems (OES) since the 1930s. In order to produce the first PRs, monocrystalline or epitaxial layers were used (Ravich, Efimova, and Smirnov 1968). However, the OES developers encountered two problems then. The first problem was that the layers with a low dark carrier concentration (preferably less than $10^{16} \mathrm{~cm}^{3}$ ) were required to increase the signal, but the technology of the pure layers was too expensive. The second problem was that in order to increase the sensitivity of PRs, the lifetime must be increased, but in extremely pure $\mathrm{PbS}$ crystals the value of $\tau$ did not exceed $10 \mu$ s.

A special feature of the impulse OES is the fact that the main criterion for the PR quality is not the increment of conductivity $\Delta \sigma$ under the influence of radiation, but the ratio $\Delta \sigma / \sigma_{0}$ divided by the effective radiation power at the fixed modulation frequency $F_{e}$. Here $\sigma_{0}$ is the dark conductivity of PR. Therefore, while the values $\tau$ and $\sigma_{0}$ are being simultaneously changed, their ratio remains constant.

It was believed that the recombination processes of the nonequilibrium carriers produced by IR radiation occur mainly according to the Auger process, namely: $\tau p^{2}=(2 \ldots 3) \cdot 10^{27} \mathrm{~cm}^{-6} \cdot \mathrm{s}$ for $\mathrm{PbS}$ and $\tau n^{2}=4 \cdot 10^{27} \mathrm{~cm}^{-6} \cdot \mathrm{s}$ for PbSe (Baryshev 2000).
The carrier lifetime in a number of the bulk PbS crystals was measured by T.S. Moss (1953) who managed to obtain the values for the lifetime in the range from $6 \cdot 10^{-10}$ to $6 \cdot 10^{-6} \mathrm{~s}$. In the early $1950 \mathrm{~s}$, R.A. Smith (1954) showed that the lifetime of one type of the carrier (electrons or holes) can be extended, if the carriers of another type are localized inside a solid or on its surface. Later, A. Rose (1963) introduced the concept of "attachment level". Creating the system consisting of small crystallites, for which the surface-tovolume ratio is much higher than the one for a single crystal, was a good idea. Therefore, presently there are a large number of $\mathrm{PbS}$ - and $\mathrm{PbSe}$-based PR-making technologies applying the vacuum deposition and the chemical deposition of layers (Johnson 1984, Saloniemi 2000).

The purpose of this paper is to show the possibility of the simulation of the carrier lifetime in polycrystalline photosensitive $\mathrm{PbSe}$ - and $\mathrm{Pb}-\mathrm{Cd}-\mathrm{S}$-based layers.

It should be noted that the analysis of the PR parameters based on the lead chalcogenides demonstrates the following regularities: despite of the differences in the energy-gap widths $\left(E_{g \mathrm{PbS}}=0.4 \mathrm{eV}, E_{g \mathrm{PbSe}}=0.25 \mathrm{eV}\right.$ at room temperature), the values of the voltage sensitivity are quite close, while the effective values of the photoconduction relaxation constant differ by 20 times. Namely, there are $15 \mu$ s for PbSe and 200...450 $\mu$ s for $\mathrm{PbS}$. The doping of the $\mathrm{PbS}$ films by halogens (I, $\mathrm{Br})$ leads to decreasing the time constant of the PbSbased structures up to $15 \mu$ s or less (Maskaeva 2004). The integration of the lead and cadmium sulfides, which are the substitutional solid solutions $\left(E_{g \mathrm{CdS}}=2.42 \mathrm{eV}\right.$ at room temperature), in various proportions allows to conduct broad changes in the energy-gap width of the $\mathrm{Pb}_{x} \mathrm{Cd}_{y} \mathrm{~S}$ material. These polycrystalline films are obtained by the hydrochemical deposition from the aqueous solutions (Markov, Maskaeva, and Ivanov 2006) as well as by the thermal evaporation 
(Aleksandrova, Maksimov, Moshnikov and Chesnakova 2008).

\section{THE PHOTOSENSITIVE FILM STRUCTURE}

In the case of substitutional solid solutions, the prepared films have varying composition in thickness. The solid solution based on the lead sulfide is formed at the depth of up to $40 \mathrm{~nm}$ approximately, while the solid solution based on the cadmium sulfide is formed at the depth of from 70 to $200 \mathrm{~nm}$. In addition, there is a transition region with a thickness of 40-70 nm (Forostyanaya, 2015). During the transition from the $\mathrm{PbS}$ to the $\mathrm{Cd}_{x} \mathrm{~Pb}_{1-x} \mathrm{~S}$ the conductivity type changes from " $n$ " to " $p$ ", while the carrier concentration decreases by 3-5 orders of magnitude with the time constant $\tau$ decreasing up to $15-60 \mu \mathrm{s}$

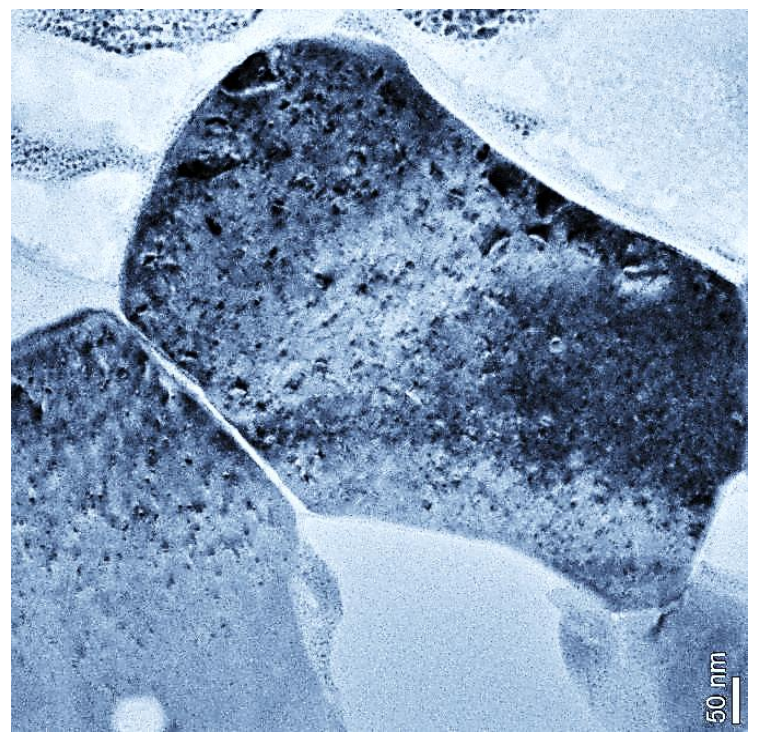

a)

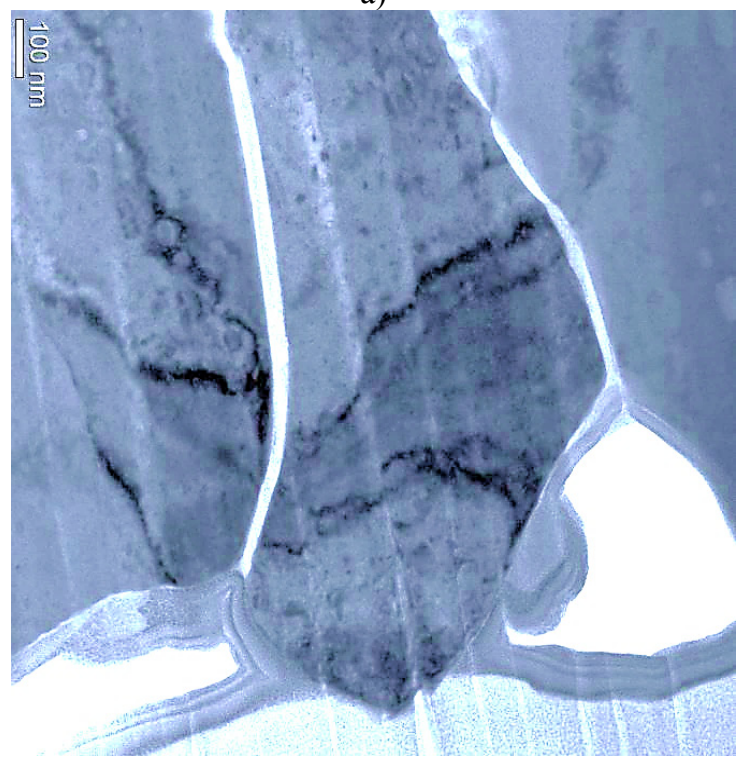

b)

Figure 1: The microphotographs of the cross-section of the physically (a) and chemically (b) deposited PbSbased photoresistors obtained with the help of the highresolution transmission electron microscopy
Due to the low carrier mobility in polycrystalline films $\left(1 \mathrm{~cm}^{2} /(\mathrm{V} \cdot \mathrm{s})\right.$ as it is stated by Petritz (1958)), the sensitivity of PR can be characterized by the ratio $\tau / p_{0}$ where $p_{0}$ is the dark carrier concentration.

The desire to increase the sensitivity of devices by increasing the carrier lifetime and to decrease the dark conductivity $\sigma_{0}$, led to the development process of PR "sensitizing". For this purpose, in the case of the PbSbased structures, oxygen was introduced into the polycrystalline film (Butkevich, Globus and Zalevskaya 1999), that is, either the high-temperature annealing in case of the physical deposition or the addition of the additives in the process of the chemical deposition, while for the PbSe-based structures, oxygen, sulfur, selenium and halogens were used (Humphrey and Scanlon 1957).

The process of conduction in the polycrystalline layers of the lead chalcogenides is greatly influenced by the intergranular barriers as well as by grain structure and size. Therefore, the study of the film morphology is of a particular importance. In Figs. 1 (Mohamed, AbdelHafiez, Miroshnikov, Barinov and Miroshnikova 2014) and 2 (Miroshnikova, Miroshnikov, Presnyakov and Mohamed 2018), the microphotographs are presented of the cross-section of the $\mathrm{PbS}$ - and $\mathrm{PbSe}$-based structures, respectively, obtained with the help of the highresolution transmission electron microscopy (HRTEM STEM/TEM Titan 80-300). In these photographs, the light keys belong to the light elements (primarily, oxygen), the dark color shows the atoms with the big numbers (sulfur, selenium, lead and iodine). It is known (Maraeva, Moshnikov and Tairov 2013) that iodine being a catalyst provides a high sensitivity for the $\mathrm{PbSe}$ based PRs due to the penetration of oxygen into the grain, which originally had $n$-type conductivity.

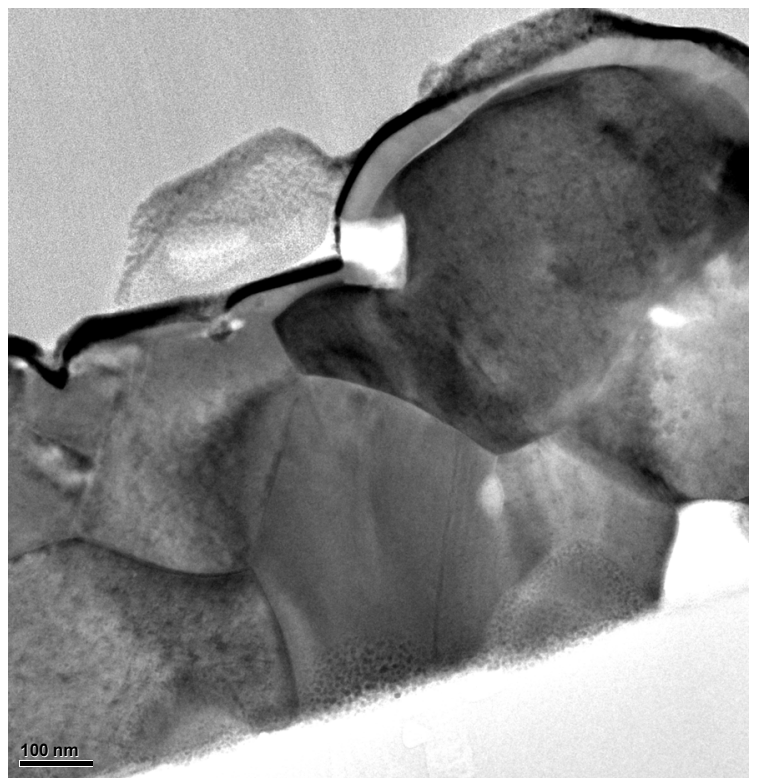

Figure 2: The microphotograph of the cross-section of the chemically deposited PbSe-based photoresistor obtained with the help of the high-resolution transmission electron microscopy 
In contrast with the PbSe-based film, in the PbS-based film one can clearly see the oxygen-containing impurities (the term introduced by Butkevich, Globus and Zalevskaya (1999)) at the crystallite boundaries. The introduction of oxygen into the $\mathrm{PbS}$ structure leads to the formation of acceptor states in the forbidden band. This, in turn, increases the hole lifetime up to 600 $\mu \mathrm{s}$, as there takes place the change of the conductivity from $n$ - to $p$-type. The dark conductivity $\sigma_{0}$ will also increase in this case.

Based on the analysis of the PR structure, one can presuppose that there are two mechanisms for the overcompensation of the $n$-type conductivity in the lead chalcogenides. Namely, in $\mathrm{PbS}$, the acceptor levels are formed both inside the grain (due to the oxygen diffusion) and on their surface (due to the oxygencontaining impurities), while for $\mathrm{PbSe}, \mathrm{PbCdS}$ the enhanced oxygen diffusion mainly occurs with iodine and bromine acting as the catalysts for this process. Oxygen as an isoelectron impurity replaces sulfur, while in the $\mathrm{PbCdS}$ substitutional solid solution it can have a low concentration of $10^{18} \mathrm{~cm}^{-3}$, approximately.

\section{SIMULATION OF THE PHOTORESISTIVE STRUCTURES RELAXATION TIME}

The photoresistive structures relaxation time is determined by the majority-carrier lifetime. As it was shown by Miroshnikova, Miroshnikov, Presnyakov, Mohamed (2018), in PbS, the concentration of holes released due to the electron trapping by the oxygencontaining impurities increases linearly with the number of the oxygen-containing impurities (the dependence $p_{2}(\tau)$ in Fig. 3a) by the law $p_{2}=1.4 \cdot 10^{20} \tau$, while the recombinations by the Auger process leads to the carrier concentration decreasing.

The influence of iodine in $\mathrm{PbSe}$ manifests itself in the stronger dependence $p_{2}=2 \cdot 10^{21} \tau$ (Fig. 3b), but in this case there also occurs the overcompensation of the conductivity type ( $n-p_{2}$ curve in Fig. $\left.3 b\right)$. As a result, one has a very bounded area of the acceptable values of the carrier lifetime in $\mathrm{PbSe}$ that provide the low dark carrier concentration.

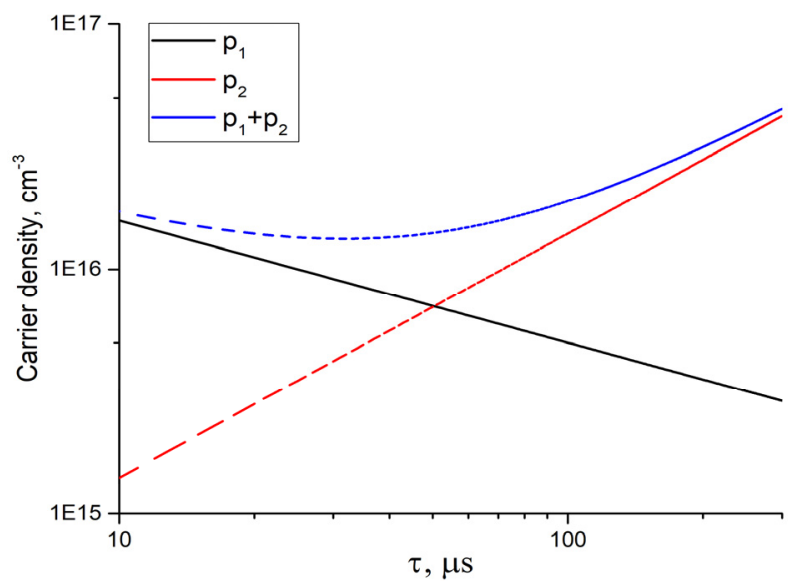

a)

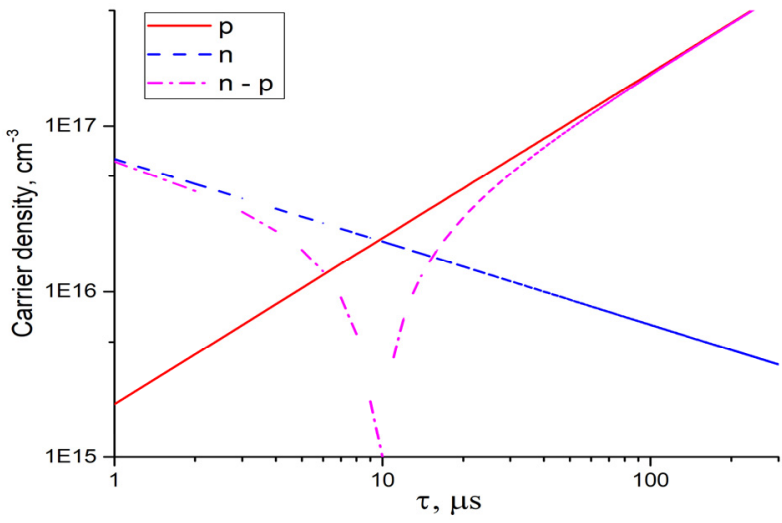

b)

Figure 3: The time dependences of the carrier concentration in the structures based on $\mathrm{PbS}$ (a) and $\mathrm{PbSe}(\mathrm{b})$

In $\mathrm{Cd}_{x} \mathrm{~Pb}_{1-x} \mathrm{~S}$ substitutional solid solution, the oxygen adsorbed by the surface is of a great significance. Our studies using the EDX and AES methods have shown that oxygen is fixed on the surface in the area of lead sulfide. Adsorbed oxygen also creates acceptor traps decreasing the dark resistance.

\section{CONCLUSION}

Thus, it has been shown that in the case of $\mathrm{PbS}$, there occurs a large variation of $\tau$ values acceptable for making the high sensitivity PRs. At the same time, in the case of $\mathrm{PbSe}$ and $\mathrm{Cd}_{x} \mathrm{~Pb}_{1-x} \mathrm{~S}$ substitutional solid solution the area of the acceptable values of the carrier lifetime becomes very bounded due to the superposition of the two processes: the overcompensation of the conductivity polarity and the high rate of the oxygen diffusion deep into the microcrystallites.

\section{ACKNOWLEDGMENTS}

This research was financially supported by the Russian Foundation for Basic Research (research project No. 1829-11051), the Ministry of Education and Science of the Russian Federation (research project No. 2.3208.2017/4.6), and the Council on grants of the President of the Russian Federation (research project No. SP-834.2019.3).

\section{REFERENCES}

Ravich Y.I., Efimova B.A. and Smirnov I.A., 1968. Methods for the study of semiconductors as applied to $\mathrm{PbTe}, \mathrm{PbSe}, \mathrm{PbS}$ lead chalcogenides [in Russian]. Moscow: Nauka.

Baryshev N.S., 2000. The properties and the application of the narrow-band-gap semiconductors [in Russian]. Kazan': Unipress.

Moss T.S., 1953. Photoelectromagnetic and photoconductive effect in lead sulphide single crystals. Proceedings of the Physical Society, B66 (12), 993-1002.

Smith R.A., 1954. The electronic and optical properties of lead sulphide group of semiconductors. Physica, 20 (11), 910-929. 
Rose A., 1963. Consepts in photoconductivity and allied problems. New York: Wiley.

Johnson T.H., 1984. Lead salt detectors and arrays $\mathrm{PbS}$ and PbSe. Proceedings of SPIE 443, pp. 60-94. August 23-25, San Diego (California, USA).

Saloniemi H., 2000. Electrodeposition of PbS, PbSe and PbTe thin films. VTT Publications 423. Espoo: Technical Research Centre of Finland.

Maskaeva L.N., 2004. Hydrochemical synthesis, structure and properties of the films of the supersaturated substitutional solid solutions $\mathrm{Me}_{\mathrm{x}} \mathrm{Pb}_{1-\mathrm{x}} \mathrm{S}$ (Me-Zn, Cd, Cu, Ag) [in Russian]. Thesis (D.Sc.). Ural State Technical University.

Markov V.F., Maskaeva L.N. and Ivanov P.N., 2006. Hydrochemical deposition of the metal sulfide films: simulation and experiment [in Russian]. Ekaterinburg: Ural Branch of RAS.

Aleksandrova O.A., Maksimov A.I., Moshnikov V.A. and Chesnakova D.B., 2008. Chalcogenides and oxides of the elements of group IV. Obtaining, study, application [in Russian]. St.-Petersburg: Technolit.

Forostyanaya N.A., 2015. Physico-chemical mechanisms of obtaining the solid solutions in the PbS-CdS system by means of the ion-exchange transformations [in Russian]. Thesis (PhD). Ural Federal University.

Petritz R.L., 1958. Theory of an experiment for measuring the mobility and density of carriers in the space-charge area of a semiconductor surface. Physical Review, 110 (6), 1254-1262.

Butkevich V.G., Globus E.R. and Zalevskaya L.N., 1999. Change of the lead sulfide films characteristics by variations of the conditions of chemically deposition from a solution. Applied Physics, (2), 52-56.

Humphrey J.N. and Scanlon W.W., 1957. Photoconductivity in lead selenide. Experimental. Physical Review, 105 (2), 469-475.

Mohamed H.S.H., Abdel-Hafiez M., Miroshnikov B.N., Barinov A.D. and Miroshnikova I.N., 2014. Spectral characteristics and morphology of nanostructured $\mathrm{Pb}-\mathrm{S}-\mathrm{O}$ thin films synthesized via two different methods. Journal of Materials Science in Semiconductor Processing, 27, 725732.

Miroshnikova I.N., Miroshnikov B.N., Presnyakov M.Y. and Mohamed H.S.H., 2018. The influence of the structure morphology on the relaxation time constant of the lead chalcogenide photoresistors [in Russian]. Proceedings of XXV International Scientific and Technical Conference on Photoelectronics and Night Viewing Devices, vol. 2, pp. 588-591. May 24-26, Moscow (Russia).

Maraeva E.B., Moshnikov V.A. and Tairov Y.M., 2013. The models of oxide phases formation in lead chalcogenide nanostructured layers obtained in oxygen and iodine vapors. Semiconductors or Physics of the Solid State, 47 (10), 1431-1434.

\section{AUTHORS BIOGRAPHY}

Irina N. Miroshnikova was born in 1957. She received the Doctor of Technical Sciences degree from the National Research University "MPEI" in 2005. Irina N. Miroshnikova is working as a Director of the Institute of Radio Engineering and Electronics and a Head of the Department of Electronics and Nanoelectronics of the National Research University "MPEI". She also holds position of Principle Research Scientist at the Institute of Nanotechnologies of Microelectronics of the Russian Academy of Science. Her main interests are physics and technology of infrared photodetectors based on singleand polycrystals.

Boris N. Miroshnikov was born in Moscow in 1990 . He received his Ph.D. in semiconductor physics from the National Research University "MPEI" in 2016. Boris N. Miroshnikov is currently working as an Assistant Professor at the Department of Electronics and Nanoelectronics of the National Research University "MPEI" as well as a Researcher at the Institute of Nanotechnologies of Microelectronics of the Russian Academy of Science. His main interests are physics and technology of infrared photodetectors based on single- and polycrystals.

Elena V. Zenova was born in 1966. She received the Doctor of Technical Sciences degree from the Russian Scientific and Research Institute of the Cable Industry in 2013. Presently, she is working as a Head of Laboratory of the Department of Development and Research of Micro- and Nanosystems of the Institute of Nanotechnologies of Microelectronics of the Russian Academy of Science as well as a Professor at the Department of Nanotechnology Microelectronics of the National Research University "MPEI". Her research interests include physics and technology of nanoelectronic devices and systems.

Mikhail Y. Presnjakov was born in 1988. He received his Ph.D. in semiconductor physics from the National Research University "MPEI" in 2014. Mikhail Y. Presnjakov is currently working as a Head of Probe and Electron Microscopy Resource Center at the National Research Center "Kurchatov Institute". His main interests are application of transmission electron microscopy methods for materials research.

Alexander A. Makarov was born in 1993. He received the B.Sc. and the M.Sc. degrees from the National Research University "MPEI" in 2014 and 2016, respectively, both in radio engineering. Presently, he is a PhD student in the Department of Electronics and Nanoelectronics at the National Research University "MPEI" as well as a Researcher at the International Laboratory of Statistics of Stochastic Processes and Quantitative Finance of the National Research Tomsk State University. His research interests include synthesis, analysis and simulation of the processing algorithms of stochastic processes and fields with unknown parameters. 